\title{
Muscular strength measurements indicate bone mineral density loss in postmenopausal women
}

This article was published in the following Dove Press journal:

Clinical Interventions in Aging

24 October 2013

Number of times this article has been viewed

\author{
Zhixiong Zhou ${ }^{1,2}$ \\ Lu Zheng ${ }^{3}$ \\ Dengyun $\mathrm{Wei}^{4}$ \\ Ming $\mathrm{Ye}^{3}$ \\ Xun $\mathrm{Li}^{2}$
}

'School of Physical Education and Coaching Science, Capital University of Physical Education and Sports, Beijing, People's Republic of China; ${ }^{2}$ Graduate School, Beijing Sport University, Beijing, People's Republic of China; ${ }^{3}$ School of Kinesiology and Health Education, Capital University of Physical Education and Sports, Beijing, People's Republic of China; ${ }^{4}$ Department of Physical Education, Anhui Normal University, Anhui, People's Republic of China
Correspondence: Zhixiong Zhou School of Physical Education and Coaching Science, Capital University of Physical Education and Sports, Beijing, People's Republic of China Tel +86 I355 2505679

Fax +86 1082099047 Email zhouzhixiongmedsci@।26.com; zhoul26@I26.com

Lu Zheng

School of Kinesiology and Health

Education, Capital University of Physical Education and Sports, Level I3, Building A3 No.9 Chegongzhuang Avenue, Beijing, People's Republic of China Email zhenglu@cupes.edu.cn
Background: The literature is inconsistent and inconclusive on the relationship between bone mineral density (BMD) and muscular strength in postmenopausal women.

Objective: To evaluate the relationship between isokinetically and isometrically determined muscle strength and BMD in postmenopausal women of different age groups.

Methods: Healthy postmenopausal women ( $n=293$; mean age, $54.22 \pm 3.85$ years) were enrolled in this study. They were grouped by age according to World Health Organization life expectancy: 45-50 years, 51-53 years, 54-56 years, 57-59 years, and 60-64 years. Total BMD, L2-4 BMD, and femoral neck BMD were measured by dual-energy X-ray bone densitometry; isokinetic and isometric muscle strength of the right hip and trunk muscles were measured during contractile exercise. Stepwise regression analysis was used to examine the relationships between BMD and strength measures, controlling for subject age and years since menopause.

Results: Results of stepwise regression showed that hip extensor and flexor strength at $120 \%$ second and back extend strength at $30 \%$ second accounted for $26 \%$ total BMD variance among menopausal subjects, 19\% L2-4 BMD variance, and 15\% femoral neck BMD variance; in postmenopausal women of different age groups, hip extensor and flexor strength at $120 \%$ second and back extend strength at $30 \%$ second accounted for $25 \%-35 \%$ total BMD variance.

Conclusion: Different optimal strength measurements were identified for different age groups. Age-appropriate testing mode can improve detection of osteoporotic fracture risk in early menopause by determining muscular strength reduction related to BMD loss. This may enable early initiation of preventative therapies.

Keywords: osteoporosis, fracture, bone mineral density, postmenopausal, menopause, muscle strength, isokinetic, isometric

\section{Introduction}

In menopausal women, changes in hormonal balance, particularly declining estrogen levels, and reduced activity result in reduced muscle strength and reduced bone mineral density (BMD). ${ }^{1}$ Although these changes are widely recognized as clinically significant because of their link with increased risk for osteoporotic fracture of the hip, lumbar spine, and lower arm, conflicting evidence exists pertaining to the relationship between muscular strength decline indicated by isometric and isokinetic testing and the declining levels of BMD. ${ }^{1-3}$ Some studies report little or no association between these factors, attributing loss of muscular strength predominantly to the increasingly sedentary lifestyle adopted by older individuals. ${ }^{3}$ Other studies report a strong concomitant decline in both muscle strength and BMD, suggesting these levels are closely and progressively related with the physiology of advancing age. ${ }^{1}$ Furthermore, recent studies have 
indicated that the most commonly employed risk assessment tools for osteoporotic fracture in aging individuals, including the World Health Organization Fracture Risk Algorithm, are increasingly inaccurate for screening risk for osteoporotic fracture. ${ }^{4,5}$ Thus, determination of the relationship between isometric and isokinetic muscle strength and BMD decline at an individual's age is paramount for development of more effective and accurate clinical instruments that assess osteoporotic fracture risk in postmenopausal women.

Estrogen levels decline progressively in postmenopausal women and cause a state of negative metabolic balance in which bone resorption often exceeds bone formation, thereby decreasing bone mass. ${ }^{6}$ In women, it has been reported that the first significant periods of muscular strength and BMD decline occur in the fifth and sixth decades of life, although more pronounced BMD declines are reported in the seventh and eighth decades. ${ }^{1}$ In the recent past, hormone replacement therapy was widely prescribed in an attempt to mediate these changes. However, use of hormone therapy has declined dramatically in the past decade because of increased concerns about adverse effects and cancer development. ${ }^{7,8}$ However, this decline in hormone replacement therapy has also raised the risk for osteoporosis and related fractures, although these therapies were previously shown to become less effective in an age-dependent manner. ${ }^{9}$ Thus, there is an increasing need for novel techniques that can detect the risk for osteoporotic fracture as individuals age, particularly with careful consideration for age groups by decade or, even more specifically, within decades.

Muscle strength has been suggested as a predictor of BMD, indirectly indicating osteoporotic fracture risk resulting from the relationship between muscle attachment site integrity and the bone's ability to withstand the biomechanical forces involved in muscular pulling. . $^{3,10,11}$ Previous reports have demonstrated a relationship between hip abductor strength and femoral BMD, as well as isokinetic knee and elbow flexor and extensor strength with vertebral and femoral BMD. ${ }^{1,3}$ In addition, reports have indicated either no relationship or a marginally significant relationship between grip strength and BMD loss. ${ }^{3}$ Furthermore, advanced age significantly delays reaction times and prolonged movement speeds, although whether these losses in motor function result from physiological changes related to aging or sedentary lifestyles of older individuals remains debatable. ${ }^{12}$

Because pathological signs are sometimes limited to certain velocity ranges, muscle strength assessments are most accurately performed by isokinetic dynamometry at various velocity spectrums. ${ }^{13}$ A recent study with trained postmenopausal women found that power training, or exercises involving high-velocity muscle shortening, were significantly correlated with maintenance of BMD, producing more effective outcomes than high-load strength training. ${ }^{14}$ Thus, there may be a measureable relationship between muscle strength decline in aging individuals and BMD loss, an important factor in osteoporosis risk that can be mediated by proper exercise or treatment regimens. However, the broad discrepancies in study populations, age groups, modes of muscle strength measurements (isometric or concentric), and testing speeds makes the published findings on these relationships arbitrary and difficult to compare.

The current study was designed to examine the relationships between age-associated muscle strength decline in the hip and trunk and loss of BMD of the total body, as well as of the hip and lumbar regions, in postmenopausal women. Muscle strength was assessed at both isometric and various concentric speeds. Findings from the study may provide clinically important data for establishing guidelines for osteoporosis risk assessment during the first decades after menopause.

\section{Materials and methods Study subjects and design}

A total of 325 healthy postmenopausal women (age range, 47-64 years) were recruited from the community to voluntarily participate in a prospective study of BMD and muscle strength that took place from May 2011 to March 2012 at a single hospital facility. Of these 325 women, 293 met the following inclusion criteria: women who had undergone menopause 1 year or more before the start of the study, women aged 47-64 years at the time of enrollment, and women who consented to all aspects of the study protocol. Exclusion criteria were women who were currently or previously administered estrogen replacement therapy or anti-osteoporotic agents, who had experienced femoral and lumbar fractures or persistent low back pain or had a history of osteoporotic fracture, who were currently taking prescribed calcium or vitamin D nutritional supplements, who had a genetically confirmed family history of metabolic diseases, who presently exhibited hypertension, who had been treated with glucocorticoids within the year before the study, or who were diagnosed with other endocrine diseases, such as diabetes, thyroid disease, kidney diseases, and tumors. The study was approved by the Ethics Committee of the Capital University of Physical Education and Sports (Beijing, People's Republic of China). All participants provided written informed consent. 


\section{Study measurements \\ Subject information}

Subjects' height and weight were measured while they were wearing light clothes and no shoes. Subjects provided their age and years since menopause.

\section{BMD assessments}

Total BMD (TBMD), L2-4 BMD, and femoral neck BMD, as well as lean body mass, were measured by dual-energy X-ray bone densitometry (Prodigy; GE, Fairfield, CT, USA). Participants were placed in the supine position with the femur rotated inward. All measurements were conducted by the same investigator.

\section{Isokinetic and isometric muscle strength determination}

To assess right hip muscle and trunk muscle strength, participants were placed in the supine position with the trunk on the measuring bench and the right thigh strapped to the lever arm of the dynamometer, or placed with both knees fixed and feet flat on the measuring bench, respectively. Isokinetic muscle strength $(\mathrm{N} \cdot \mathrm{m})$ of the right hip and trunk muscles was measured with an ISOMED2000 isokinetic system (Basic System and Back System; D\&R Ferstl GmbH, Hemau, Germany). Three to eight consecutive measurements of maximum peak torque were taken, as previously described. ${ }^{14}$ An appropriate testing mode (isometric or isokinetic) and speed were selected to maximize data collection. ${ }^{13}$ Isometric contractile strengths of the hip extensor at $90^{\circ}$, hip flexors at $20^{\circ}$, and back extensor/ flexors at $20^{\circ}$ were measured. Isokinetic concentric contractile strength by angular velocity of $120 \%$ second and $180 \%$ second for the hip extensor/flexor and $30 \%$ second, $60^{\circ} /$ second, and $120 \%$ second for the trunk extensor/flexor were measured. Notably, trunk extensor and flexor measurements were only conducted using the isokinetic contractile strength because of the well-recognized reliability of these measurements for the trunk region. ${ }^{16}$ Corrections were made for gravity. ${ }^{14}$

\section{Statistical analyses}

We used power analysis to determine an adequate sample size. According to Humphries et al, ${ }^{17}$ the correlation coefficient $(r)$ for isokinetic muscle strength of lower back muscles and isokinetic peak torque of back extensor muscles at a speed of $30 \%$ second, and that for L2-4 BMD in postmenopausal women, is $r=0.21$. To detect a simple correlation $r(r=0.21-0.5)$, using a two-sided test and a $5 \%$ significance level test $(\alpha=0.05)$ with a power of $80 \%(\beta=0.2)$, the required sample size was approximately $29-176$.
Analysis of variance with Duncan's multiple range post hoc tests was performed to examine the age-related trend on BMD and muscle strength measures. Participants were grouped by ages 50 years or younger, $51-53$ years, 54-56 years, 57-59 years, and 60 years or older, based on life expectancy ranges provided by the World Health Organization Guidelines for Life Expectancy. ${ }^{15}$ Associations between muscle strength and BMD were explored by Pearson's correlations and partial correlations controlling for age. Stepwise multiple regression on BMD measurements was used to identify significant predictive variables from measures of muscle strength of different testing modes and muscle groups, after controlling for age and years since menopause. Trend analysis was performed to identify significant predictive variables from each testing mode (isometric or isokinetic) for trunk extensor and flexor measurements to avoid confounding from having too many strength variables in regression models. Participant age and years since menopause were entered in the first step of the regression. Muscle strength variables were entered and retained if they met inclusion criteria $(P<0.05)$. The same analysis was repeated with age stratification (age groups, $\leq 50,51-53$, $54-56,57-59$, and $\geq 60$ years). $P$-values $\leq 0.05$ (two-sided) were considered statistically significant in all analyses. All data were recorded as mean values \pm standard deviations (SD) and analyzed using SPSS version 18.0 (IBM Corporation, Armonk, NY, USA).

\section{Results}

Table 1 displays the data collected from these women (mean age, $54.22 \pm 3.85$ years). A total of 56, 79, 81, 56, and 21 participants were included in the $45-50$ years, $51-53$ years, 54-56 years, 57-59 years, and 60 years and older age groups, respectively. The mean time after menopause was $5.34 \pm 3.53$ years (range, 1-17 years), mean body weight was $62.27 \pm 7.68 \mathrm{~kg}$, and mean body mass index was $25.20 \pm 2.91 \mathrm{~kg} / \mathrm{m}^{2}$. There were significant trends of decline in body weight, body mass index, total lean mass, and BMD measurements, and the majority of strength measurements across the participants' age groups $(P<0.05)$, with the exception of height and total fat mass.

\section{BMD and muscle strength by age groups}

Coefficients of variation (CV) for BMD were estimated at $0.66 \%$ for TBMD, $0.93 \%$ for $\mathrm{L} 2-4,0.44 \%$ for total hip, and $0.79 \%$ for the femoral neck. The CV values for fat mass and lean body mass were $1.69 \%$ and $0.52 \%$, respectively. BMD measurements declined with age. Duncan's multiple 
Table I Demographics, body composition, and strength measures by subject age group $(\mathrm{N}=293)$

\begin{tabular}{|c|c|c|c|c|c|c|}
\hline \multirow[t]{2}{*}{ Characteristic } & \multirow[t]{2}{*}{ All $(n=293)$} & \multicolumn{5}{|l|}{ Age, years } \\
\hline & & $\leq 50(n=56)$ & $5 I-53(n=79)$ & $54-56(n=8 I)$ & $57-59(n=56)$ & $\geq 60(n=21)$ \\
\hline Subject age* & $54.22 \pm 3.85$ & $48.64 \pm 1.19$ & $52.42 \pm 0.82$ & $55.23 \pm 0.91$ & $58.22 \pm 0.86$ & $61.39 \pm 1.25$ \\
\hline Postmenopausal years* & $5.34 \pm 3.53$ & $2.33 \pm 1.21$ & $3.86 \pm 2.39$ & $5.74 \pm 3.02$ & $8.05 \pm 3.39$ & $10.12 \pm 2.86$ \\
\hline Height & $157.19 \pm 5.04$ & $157.59 \pm 5.12$ & $156.13 \pm 5.07$ & $157.62 \pm 5.03$ & $|57.20 \pm 5.1|$ & $158.43 \pm 4.26$ \\
\hline Weight* & $62.27 \pm 7.68$ & $63.97 \pm 8.51$ & $62.57 \pm 7.52$ & $61.85 \pm 7.78$ & $61.74 \pm 6.56$ & $59.64 \pm 8.00$ \\
\hline Body mass index, $\mathrm{kg} / \mathrm{m}^{2 *}$ & $25.20 \pm 2.91$ & $25.76 \pm 3.20$ & $25.67 \pm 2.88$ & $24.88 \pm 2.78$ & $25.00 \pm 2.56$ & $23.75 \pm 3.08$ \\
\hline Total fat mass & $22.08 \pm 5.05$ & $21.94 \pm 5.67$ & $22.64 \pm 4.45$ & $22.01 \pm 5.21$ & $21.94 \pm 4.39$ & $21.01 \pm 6.47$ \\
\hline Total lean mass* & $36.85 \pm 3.51$ & $38.17 \pm 3.35$ & $36.4 I \pm 3.5 I$ & $36.39 \pm 3.45$ & $36.91 \pm 3.67$ & $36.62 \pm 3.01$ \\
\hline \multicolumn{7}{|l|}{ BMD } \\
\hline Total body* & $1.07 \pm 0.09$ & $1.14 \pm 0.09$ & $1.09 \pm 0.08$ & $1.05 \pm 0.08$ & $1.05 \pm 0.08$ & $\mathrm{I} .0 \mathrm{I} \pm 0.08$ \\
\hline Lumbar $2-4^{*}$ & $1.10 \pm 0.16$ & $1.20 \pm 0.16$ & $1.11 \pm 0.15$ & $1.05 \pm 0.14$ & $1.08 \pm 0.15$ & $0.99 \pm 0.11$ \\
\hline Femoral* & $0.93 \pm 0.12$ & $0.99 \pm 0.15$ & $0.94 \pm 0.10$ & $0.92 \pm 0.12$ & $0.90 \pm 0.12$ & $0.84 \pm 0.08$ \\
\hline Femoral neck* & $0.87 \pm 0.12$ & $0.93 \pm 0.14$ & $0.89 \pm 0.11$ & $0.85 \pm 0.11$ & $0.83 \pm 0.11$ & $0.79 \pm 0.07$ \\
\hline \multicolumn{7}{|l|}{ Hip } \\
\hline Extend isometric, $90^{\circ}$ & $186.12 \pm 46.0$ & $184.57 \pm 48.19$ & $|88.4| \pm 52.77$ & $189.85 \pm 43.45$ & $|79.54 \pm 35.5|$ & $\mid 84.81 \pm 49.22$ \\
\hline Flex isometric, $20^{\circ} *$ & $58.72 \pm 15.75$ & $60.95 \pm 16.15$ & $58.81 \pm 14.84$ & $61.81 \pm 15.48$ & $54.63 \pm 15.58$ & $51.38 \pm 16.27$ \\
\hline Flex, $120 \%$ second* & $45.25 \pm 10.26$ & $48.98 \pm 10.92$ & $44.51 \pm 10.83$ & $44.64 \pm 9.59$ & $44.30 \pm 10.38$ & $42.90 \pm 5.96$ \\
\hline Extend, $120 \%$ second & $107.18 \pm 27.65$ & $106.91 \pm 30.34$ & $110.25 \pm 27.70$ & $105.12 \pm 26.50$ & $105.32 \pm 25.98$ & $109.24 \pm 30.09$ \\
\hline Flex, $180^{\circ} /$ second $*$ & $54.28 \pm 14.23$ & $58.41 \pm 14.44$ & $55.75 \pm 13.82$ & $53.54 \pm 14.39$ & $50.11 \pm 14.47$ & $51.76 \pm 11.02$ \\
\hline Extend, $180^{\circ} /$ second & $85.01 \pm 29.89$ & $85.20 \pm 29.53$ & $87.84 \pm 30.20$ & $81.62 \pm 29.34$ & $85.09 \pm 28.88$ & $86.81 \pm 35.70$ \\
\hline \multicolumn{7}{|l|}{ Back } \\
\hline Flex, $30 \%$ second $*$ & $68.51 \pm 21.24$ & $77.89 \pm 24.21$ & $67.53 \pm 21.54$ & $66.22 \pm 17.86$ & $65.14 \pm 21.70$ & $64.90 \pm 16.70$ \\
\hline Extend, $30^{\circ} /$ second $*$ & $136.55 \pm 35.64$ & $147.43 \pm 37.15$ & $140.33 \pm 38.20$ & $129.79 \pm 28.75$ & $138.04 \pm 31.88$ & $115.38 \pm 43.67$ \\
\hline Flex, $60^{\circ} /$ second $*$ & $59.59 \pm 20.06$ & $65.84 \pm 21.75$ & $57.24 \pm 19.88$ & $59.59 \pm 18.90$ & $57.14 \pm 20.89$ & $58.33 \pm 15.93$ \\
\hline Extend, $60 \%$ second $*$ & $117.28 \pm 40.92$ & $125.77 \pm 45.70$ & $121.22 \pm 40.89$ & $115.20 \pm 40.50$ & $111.96 \pm 38.13$ & $102.05 \pm 32.08$ \\
\hline Extend, $120 \%$ second $*$ & $60.74 \pm 24.4 I$ & $67.07 \pm 30.32$ & $59.71 \pm 24.78$ & $58.89 \pm 21.76$ & $58.25 \pm 21.76$ & $61.48 \pm 16.00$ \\
\hline Flex, $120^{\circ} /$ second* & $84.95 \pm 40.48$ & $90.16 \pm 40.23$ & $91.53 \pm 45.11$ & $83.60 \pm 38.26$ & $82.41 \pm 38.70$ & $58.29 \pm 22.49$ \\
\hline Flex isometric, $20^{\circ} *$ & $197.39 \pm 42.05$ & $200.91 \pm 40.90$ & $199.91 \pm 43.14$ & $197.26 \pm 35.99$ & $197.89 \pm 37.86$ & $177.62 \pm 66.11$ \\
\hline Extend isometric, $20^{\circ} *$ & $65.02 \pm 22.93$ & $68.34 \pm 18.36$ & $62.16 \pm 21.11$ & $67.21 \pm 29.10$ & $65.09 \pm 21.41$ & $58.33 \pm 15.19$ \\
\hline
\end{tabular}

Notes: Data are expressed as mean values \pm standard deviation. Statistical significance is represented by $* P<0.05$.

Abbreviation: BMD, bone mineral density.

range tests indicated that TBMD, L2-4 BMD, and femoral neck BMD were significantly higher in the 45-50-year age group when compared with all other age groups $(P<0.05)$. The difference between the 54-56-year age group and the 57-59-year age group was not significant $(P>0.05)$. TBMD and L2-4 BMD were significantly higher in the 51-53-year age groups when compared with the 54-56-year, 57-59-year, and $\geq 60$-year age groups $(P<0.001 ; P=0.014)$, although the femoral neck BMD did not differ significantly between these groups.

Most of isokinetic hip and trunk muscle strengths were significantly different in postmenopausal women of the five age groups $(P<0.05)$ with the exception of hip extend isometric at $90^{\circ} /$ second and hip extend at $120^{\circ}$ /second and $180 \%$ second $(P>0.05)$. Overall, significant declines of isokinetic hip and trunk muscle strengths with increasing age were observed in most angular velocities (Table 1).

\section{Correlations among BMD, hip and trunk muscle contraction strength, and speed}

Pearson's correlation coefficients between isokinetic hip and trunk muscle strengths and hip/lumbar BMD are shown in Table 2, and partial correlations controlling for age are shown in Table 3. Overall, BMD correlated with isokinetic strength at different angular velocities, with declining BMD correlating closely with reduced isokinetic strength in both the extensor and flexor muscles that was most apparent at slower speeds.

\section{Relationships between hip isometric and isokinetic extensor and flexor strength and BMD measurements}

Results of multiple stepwise regression analysis on TBMD, L2-4 BMD, femoral neck BMD, and femoral BMD showed that hip extend at $120 \%$ second, back extend at $30^{\circ}$ second, and 
Table 2 Correlations between subject age, total lean mass, muscle strength, and bone density $(\mathrm{N}=293)$

\begin{tabular}{|c|c|c|c|c|c|c|}
\hline \multirow[t]{2}{*}{ Pearson correlation } & \multirow{2}{*}{$\begin{array}{l}\text { Subject's } \\
\text { age }\end{array}$} & \multirow{2}{*}{$\begin{array}{l}\text { Total lean } \\
\text { mass }\end{array}$} & \multicolumn{4}{|l|}{ BMD } \\
\hline & & & Total body & BMD L2-4 & Femoral neck & Femoral \\
\hline Subject's age & $1.00 * *$ & 0.11 & $0.42 * *$ & $0.36 * *$ & $0.35^{* *}$ & $0.33^{* *}$ \\
\hline Total lean mass & 0.10 & 1.00 & $0.45 * *$ & $0.22^{* *}$ & $0.33^{* *}$ & $0.29 * *$ \\
\hline \multicolumn{7}{|l|}{ Hip } \\
\hline Extend isometric, $90^{\circ}$ & 0.02 & $0.40 * *$ & $0.42^{* *}$ & $0.4 I^{* *}$ & $0.34 * *$ & $0.34^{* *}$ \\
\hline Flex isometric, $20^{\circ}$ & $0.13^{*}$ & $0.34 * *$ & $0.23 * *$ & $0.20 * *$ & $0.20 * *$ & $0.14^{*}$ \\
\hline Flex, $120 \%$ second & $0.16 * *$ & $0.44 * *$ & $0.45 * *$ & $0.37 * *$ & $0.32 * *$ & $0.31 * *$ \\
\hline Extend, $120 \%$ second & 0.02 & $0.38 * *$ & $0.43 * *$ & $0.34 * *$ & $0.34 * *$ & $0.33 * *$ \\
\hline Flex, $180 \%$ second & $0.17 * *$ & $0.30 * *$ & $0.27^{* *}$ & $0.25 * *$ & $0.14 *$ & $0.17^{* *}$ \\
\hline Extend, $180 \%$ second & 0.01 & $0.25^{* *}$ & $0.17 * *$ & $0.16 * *$ & 0.09 & $0.11 *$ \\
\hline \multicolumn{7}{|l|}{ Back } \\
\hline Flex, $30 \%$ second & $0.18 * *$ & $0.33 * *$ & $0.36 * *$ & $0.38 * *$ & $0.37^{* *}$ & $0.33^{* *}$ \\
\hline Extend, $30 \%$ second & $0.20 * *$ & $0.32 * *$ & $0.48 * *$ & $0.46 * *$ & $0.38^{* *}$ & $0.39 * *$ \\
\hline Flex, $60^{\circ} /$ second & $0.12^{*}$ & $0.35^{* *}$ & $0.31 * *$ & $0.28 * *$ & $0.28 * *$ & $0.23^{* *}$ \\
\hline Extend, $60 \%$ second & $0.17 * *$ & $0.20 * *$ & $0.32 * *$ & $0.28 * *$ & $0.26 * *$ & $0.26 * *$ \\
\hline Extend, $120 \%$ second & 0.10 & 0.08 & 0.09 & $0.11 *$ & 0.06 & 0.05 \\
\hline Flex, $120 \%$ second & $0.17^{* *}$ & 0.09 & $0.17^{* *}$ & $0.20 * *$ & $0.13^{*}$ & $0.15^{* *}$ \\
\hline Flex isometric, $20^{\circ}$ & 0.08 & $0.30 * *$ & $0.28 * *$ & $0.21^{* *}$ & $0.22 * *$ & $0.21 * *$ \\
\hline Extend isometric, $20^{\circ}$ & 0.05 & $0.21^{* *}$ & $0.14^{*}$ & $0.14 *$ & $0.12 *$ & 0.08 \\
\hline
\end{tabular}

Note: Statistical significance is represented by $* * P<0.0$ I; $* P<0.05$.

Abbreviation: BMD, bone mineral density.

hip flex at $120 \%$ second accounted for $26 \%$ of the variation in TBMD, whereas hip extend at $120 \%$ second and back extend at $30 \%$ second accounted for $15 \%-19 \%$ of variance in L2-4 BMD, femoral neck BMD, and femoral BMD after controlling for age and years since menopause (Table 4 ). Back extend at $30^{\circ} /$ second had the highest partial $r$ except for femoral neck BMD. Muscle strength measures from other velocities and/or modes did not enter the regression. Participant age and menopausal years were inversely associated with BMD and also explained a significant amount of variance $(12 \%-19 \%)$ in regression models.

The results of age-group stratified stepwise multiple regression on TBMD are shown in Table 5. Hip flex at

Table 3 Partial correlations between total lean mass, muscle strength, and bone density measures after controlling for subject age $(\mathrm{N}=293)$

\begin{tabular}{|c|c|c|c|c|c|}
\hline \multirow[t]{2}{*}{ Controlling age } & \multirow{2}{*}{$\begin{array}{l}\text { Total lean } \\
\text { mass }\end{array}$} & \multicolumn{4}{|l|}{ BMD } \\
\hline & & Total body & L2-4 & Femoral neck & Femoral \\
\hline Total lean mass & 1.00 & $0.46 * *$ & $0.20 * *$ & $0.32 * *$ & $0.27 * *$ \\
\hline \multicolumn{6}{|l|}{ Hip } \\
\hline Extend isometric at $90^{\circ}$ & $0.40 * *$ & $0.45 * *$ & $0.43^{* *}$ & $0.36 * *$ & $0.35 * *$ \\
\hline Flex isometric at $20^{\circ}$ & $0.33 * *$ & $0.19 * *$ & $0.16 * *$ & $0.16 * *$ & 0.11 \\
\hline Flex at $120 \% /$ second & $0.42 * *$ & $0.43 * *$ & $0.34 * *$ & $0.28 * *$ & $0.28 * *$ \\
\hline Extend at $120 \%$ second & $0.38 * *$ & $0.47^{* *}$ & $0.36 * *$ & $0.36 * *$ & $0.35 * *$ \\
\hline Flex at $180 \%$ second & $0.28 * *$ & $0.22 * *$ & $0.21 * *$ & 0.09 & $0.12 *$ \\
\hline Extend at $180 \%$ second & $0.24 * *$ & $0.19 * *$ & $0.17 * *$ & 0.10 & $0.12 *$ \\
\hline \multicolumn{6}{|l|}{ Back } \\
\hline Flex at $30^{\circ} /$ second & $0.32 * *$ & $0.32 * *$ & $0.35 * *$ & $0.34 * *$ & $0.29 * *$ \\
\hline Extend at $30 \%$ second & $0.31 * *$ & $0.45^{* *}$ & $0.43 * *$ & $0.34 * *$ & $0.35 * *$ \\
\hline Flex at $60 \%$ second & $0.34 * *$ & $0.29 * *$ & $0.26 * *$ & $0.25 * *$ & $0.20 * *$ \\
\hline Extend at $60 \%$ second & $0.18 * *$ & $0.27 * *$ & $0.24 * *$ & $0.21 * *$ & $0.22 * *$ \\
\hline Extend at $120 \%$ second & 0.07 & 0.05 & 0.08 & 0.02 & 0.02 \\
\hline Flex at $120 \% /$ second & 0.07 & 0.11 & $0.15^{* *}$ & 0.08 & 0.10 \\
\hline Flex isometric at $20^{\circ}$ & $0.29 * *$ & $0.27 * *$ & $0.19 * *$ & $0.21 * *$ & $0.20 * *$ \\
\hline Extend isometric at $20^{\circ}$ & $0.21 * *$ & $0.13^{*}$ & $0.13^{*}$ & 0.11 & 0.06 \\
\hline
\end{tabular}

Note: Statistical significance is represented by $* * P<0.01 ; * P<0.05$.

Abbreviation: BMD, bone mineral density. 
Table 4 Unstandardized coefficient, standard error, partial correlation, and adjusted $R^{2}$ of stepwise multiple regression analysis on bone density

\begin{tabular}{|c|c|c|c|c|c|}
\hline Predictor variables & B & SE & $P$-value* & $r$ & Adjusted $R^{2}$ \\
\hline \multicolumn{6}{|l|}{ Total body BMD } \\
\hline Step I & & & & & 0.19 \\
\hline Intercept & $1.48 \mid$ & 0.0840 & 0.0001 & & \\
\hline Postmenopausal years & -0.004 & 0.0020 & 0.026 & -0.12 & \\
\hline Subject age & -0.007 & 0.0020 & 0.0001 & -0.23 & \\
\hline Step 2 & & & & & 0.26 \\
\hline Hip extend at $120^{\circ} /$ second & 0.001 & 0.0001 & 0.0001 & 0.20 & \\
\hline Back extend at $30 \%$ second & 0.001 & 0.0001 & 0.0001 & 0.23 & \\
\hline Hip flex at $120 \%$ second & 0.001 & 0.0001 & 0.009 & 0.12 & \\
\hline \multicolumn{6}{|l|}{ BMD L2-4 } \\
\hline Step I & & & & & 0.14 \\
\hline Intercept & 1.692 & 0.1520 & 0.0001 & & \\
\hline Postmenopausal years & -0.007 & 0.0030 & 0.027 & -0.12 & \\
\hline Subject age & -0.01 & 0.0030 & 0.001 & -0.18 & \\
\hline Back extend at $30 \%$ second & 0.001 & 0.0001 & 0.0001 & 0.30 & \\
\hline Step 2 & & & & & 0.19 \\
\hline Hip extend at $120 \%$ second & 0.001 & 0.0001 & 0.0001 & 0.19 & \\
\hline \multicolumn{6}{|l|}{ BMD of femoral } \\
\hline Step I & & & & & 0.12 \\
\hline Intercept & 1.339 & 0.1230 & 0.0001 & & \\
\hline Postmenopausal years & -0.006 & 0.0030 & 0.027 & -0.12 & \\
\hline Subject age & -0.007 & 0.0020 & 0.005 & -0.16 & \\
\hline Back extend at $30 \%$ second & 0.001 & 0.0001 & 0.0001 & 0.23 & \\
\hline Step 2 & & & & & 0.15 \\
\hline Hip extend at $120 \%$ second & 0.001 & 0.0001 & 0.0001 & 0.22 & \\
\hline \multicolumn{6}{|l|}{ BMD of femoral neck } \\
\hline Step I & & & & & 0.15 \\
\hline Intercept & 1.245 & 0.1170 & 0.0001 & & \\
\hline Postmenopausal years & -0.008 & 0.0030 & 0.002 & -0.17 & \\
\hline Subject age & -0.006 & 0.0020 & 0.008 & -0.14 & \\
\hline Hip extend at $120 \%$ second & 0.001 & 0.0001 & 0.0001 & 0.23 & \\
\hline Step 2 & & & & & 0.15 \\
\hline Back extend at $30 \%$ second & 0.001 & 0.0001 & 0.0001 & 0.21 & \\
\hline
\end{tabular}

Note: *All regression models were statistically significant $(P<0.05)$.

Abbreviations: $B$, unstandardized coefficient; SE, standard error; $r$, partial correlation; BMD, bone mineral density.

Table 5 Unstandardized coefficient, standard error, partial correlation, and adjusted $R^{2}$ of stepwise multiple regression on total bone density, stratified by age group

\begin{tabular}{|c|c|c|c|c|c|}
\hline $\begin{array}{l}\text { Age group in years } \\
\text { and predictor variables }\end{array}$ & B & SE & $P$-value* & $\boldsymbol{r}$ & Adjusted $R^{2 \ddagger}$ \\
\hline$\leq 50$ & & & & & 0.30 \\
\hline Hip flex at $120^{\circ} /$ second & 0.003 & 0.001 & 0.018 & 0.27 & \\
\hline Back extend at $30 \%$ second & 0.001 & 0.0001 & 0.02 & 0.27 & \\
\hline $51-53$ & & & & & 0.28 \\
\hline Back extend at $30 \%$ second & 0.001 & 0.0001 & 0.024 & 0.22 & \\
\hline Hip extend at $120 \%$ second & 0.001 & 0.0001 & 0.0001 & 0.36 & \\
\hline $54-56$ & & & & & 0.35 \\
\hline Hip flex at $120^{\circ} /$ second & 0.002 & 0.001 & 0.028 & 0.19 & \\
\hline Back extend at $30 \%$ second & 0.001 & 0.0001 & 0.001 & 0.29 & \\
\hline Hip extend at $120 \%$ second & 0.001 & 0.0001 & 0.025 & 0.19 & \\
\hline $57-59$ & & & & & 0.25 \\
\hline Hip flex at $120 \%$ second & 0.004 & 0.001 & 0.0001 & 0.52 & \\
\hline$\geq 60$ & & & & & 0.31 \\
\hline Hip flex at $120 \%$ second & 0.009 & 0.002 & 0.002 & 0.61 & \\
\hline
\end{tabular}

Notes: *All regression models were statistically significant $(P<0.05)$; ${ }^{\ddagger}$ variance accounted for by variables retained at the last step of regression, adjusted for postmenopausal years. Abbreviations: $B$, unstandardized coefficient; SE, standard error; $r$, partial correlation. 
$120 \%$ second was most consistent as a predictor across the age groups, except in the 51-53 year old group, whereas back extend at $30 \%$ second was significant in the three youngest age groups. The amount of variance accounted for by the muscle strength measures was higher in each age group compared with those in the models with all participants (Table 4). The results were similar for $\mathrm{L} 2-4 \mathrm{BMD}$, femoral neck BMD, and femoral BMD (data not shown).

\section{Discussion}

This study provided a close examination of the age-mediated relationships between muscle strength and BMD in a group of postmenopausal women. To do so, we used muscle strength measurements obtained isokinetically and isometrically over a variety of speeds for hip and trunk muscle groups. Our data show a clear, concomitant decline in muscle strength of the hip and trunk and BMD with advanced age. Although the biggest decline in muscle strength was measured in the 54-56 year old age group, the decline was most significant in participants older than 60 years. The decline in BMD occurred gradually but was most obvious in the participants aged 60 years and older. Muscle strength measurements significantly explained the variations in BMD (15\%-19\%) after controlling for age and postmenopausal years. Hip flex at $120 \%$ second and back extension at $30 \%$ second were the two most consistent predictor variables for all BMD measurements across all age groups.

This comprehensive analysis of TBMD, L2-4 BMD, and femoral neck BMD loss indicated that BMD decreased with increasing age. Furthermore, the decline of TBMD and L2-4 BMD closely correlated with reduced muscle strengths, particularly declining isometric strength of the hip extensor at $90^{\circ}$ and declining isokinetic strengths of the trunk flexor and extensor at $30 \%$ second and $60 \%$ second, in participants aged between 45-50 years. A less apparent correlation was observed in participants of more advanced ages (over 60 years old) with a longer time since initiation of menopause. Although overall hip and trunk isokinetic strengths may not exhibit a close correlation with age in many individuals, this study indicates that changes in hip and trunk muscle strengths were apparent at particular angular velocities, which may be optimal for prognostic assessment in these individuals. Although further research of wider age groups, particularly those spanning into the seventh and eighth decades of life, will be required to confirm these findings, the current results provide compelling preliminary evidence that the early menopausal period during the late fourth decade to the mid-sixth decade of life are a critical period for interrelated muscular and BMD decline. This has particular significance for routine screening and osteoporosis prevention strategies, suggesting early initiation of preventative care should begin at the onset of muscular decline, even before significant loss of BMD.

The findings that BMD and muscular strength decline with age are consistent with values reported by previous reports indicating progressive decreases in BMD between ages 40 and 79 years. ${ }^{11,18}$ Contrary to previous schools of thought, however, the decrease in BMD with age has been demonstrated to be nonlinear, necessitating improved screening strategies for prognostic prediction in postmenopausal individuals. ${ }^{19}$ Furthermore, as demonstrated by the present study, activation patterns of isometric and isokinetic, as well as dynamic, muscle actions at the same joint angle vary widely, compounding the use of muscular strength assessments in routine clinical practice. ${ }^{20}$ Notably, this study identified a correlation between BMD and isokinetic strength at different angular velocities in postmenopausal women that was closely associated with increasing age from 47 to 59 years, becoming less apparent thereafter. These findings may be potentially useful for improving the quality of routine clinical assessment of women during the early postmenopausal period, a critical time for initiation of BMD loss prevention strategies, such as strength training or other therapies, which often occurs before symptomatic presentations such as osteoporosis or osteoporotic fracture. ${ }^{14}$

The current study indicated that BMD decline correlated closely with isokinetic strength of the hip and trunk at higher angular velocities in the majority of early menopause individuals, most apparently indicating reduced TBMD and L2-4 BMD. Similar findings have been previously reported for reduced knee and quadriceps strength at $90 \%$ second, which correlated significantly with femoral neck BMD but not L2-4 BMD in postmenopausal women. ${ }^{21}$ Bayramoğlu et al observed that isokinetic strength of hip abductors correlated weakly with femoral BMD and that trunk muscle strength did not correlate with lumbar BMD in postmenopausal women, although these associations were reported to be very weak as a result of the effect of the sedentary lifestyle of older women. ${ }^{3}$ We propose, however, that these discrepancies may instead be predominantly related to variant pathological deterioration in strength at different speeds, which is consistent with the variations in functional movement speed and load-bearing reported in menopausal women by von Stengel et al. ${ }^{14}$ In line with the current results, Sherk et a ${ }^{18}$ successfully demonstrated an association between TBMD using muscle strength and fat mass, and Taaffe et $\mathrm{al}^{11}$ demonstrated that dynamic leg muscle strength was a robust predictor of BMD in elderly women. The current study, however, is the first to suggest 
that a distinct and nonlinear relationship exists between muscle strength reduction and BMD loss by age. This may be useful as a prognostic indicator of osteoporosis in addition to conventional body weight and mass assessments. ${ }^{22}$ It also implies that women may be more susceptible to progressive BMD during early menopause if they exhibit notable muscle loss during this early period.

Notably, several recent studies have suggested that the parameters demonstrated to be related to BMD in postmenopausal women using significant univariate relationships are drastically reduced after adjusting for the confounding variable of body weight. ${ }^{23,24}$ Thus, the current study further divided groups by age and testing type (isometric or isokinetic) to more clearly distinguish the most significant parameters associated with BMD. However, this increased the degrees of freedom involved with such subdivision for analytical purposes. ${ }^{25}$ Further clinical validation will be required to confirm these findings and eliminate possible confounding variables. We did, however, control for lean body mass in the current study, as was suggested in previous studies. $^{23,24}$ The univariate correlation coefficient between muscle strength and BMD significantly decreased in postmenopausal women. A significant correlation also remained between BMD and the majority of variables of isokinetic strength at other speeds, a finding confirmed by previous research. ${ }^{26}$ Thus, these findings, although requiring further clinical validation because of the inherent nature of complex statistical analyses, are highly consistent with findings of other similar studies.

We also concede that this study had several limitations. First, we did not measure plasma and estrogen levels. We excluded patients who were currently taking or who had previously been administered estrogen replacement therapy or anti-osteoporotic agents, and who had been prescribed calcium or vitamin D nutritional supplements. We believe the plasma estrogen and calcium levels in each age group represent populations that vary individually but are within the normal range. However, these factors may affect BMD or hip extensor/flexor strength and should be further examined. In addition, other important subject demographic information could have been included in our analyses, and we will consider expanding the scope of our investigations in future studies.

This study confirmed the correlation between muscle strength and BMD, using isometric and isokinetic testing modes, demonstrating an age-dependent reduction in BMD and muscle strength throughout early menopause. These findings provide a reference for the selection of appropriate modes of testing and angular velocities for muscular strength of the hip and trunk, providing a basis for potential future development of clinical prognostic tests capable of indicating BMD loss and osteoporosis risk. Furthermore, clinical assessments based on these parameters may have greater utility for detecting osteoporotic fracture risk with high anatomical specificity, thereby accurately indicating the bones or joints most prone to failure rather than conventional risk by patient characteristics. Further study, however, will be required to clinically confirm the validity of the correlations between BMD loss and reduced muscle strength determined in this study before widely applicable clinical guidelines can be generated.

\section{Acknowledgments}

This study was supported by the Beijing Municipality Project for Academic Human Resources Development in Institutions of Higher Learning (PHR-IHLB2007-17). Funding was provided by "National Key Technology Research and Development Program of China during the 12th Five-year Plan Period" (No 2012BAK21B00). The study protocol was approved by Capital University of Physical Education and Sports Ethics Committee, Beijing, People's Republic of China (Approval ID: XJYYLL-2007002).

\section{Disclosure}

The authors report no conflicts of interest in this work.

\section{References}

1. Calmels P, Vico L, Alexandre C, Minaire P. Cross-sectional study of muscle strength and bone mineral density in a population of 106 women between the ages of 44 and 87 years: relationship with age and menopause. Eur J Appl Physiol Occup Physiol. 1995;70(2):180-186.

2. Pal L, Hailpern SM, Santoro NF, et al. Increased incident hip fractures in postmenopausal women with moderate to severe pelvic organ prolapse. Menopause. 2011;18(9):967-973.

3. Bayramoğlu M, Sözay S, Karataş M, Kilinç S. Relationships between muscle strength and bone mineral density of three body regions in sedentary postmenopausal women. Rheumatol Int. 2005;25(7):513-517.

4. Sandhu SK, Nguyen ND, Center JR, Pocock NA, Eisman JA, Nguyen TV. Prognosis of fracture: evaluation of predictive accuracy of the FRAX algorithm and Garvan nomogram. Osteoporos Int. 2010;21(5):863-871.

5. Kaunitz AM, McClung MR, Feldman RG, Wysocki S. Postmenopausal osteoporosis: fracture risk and prevention. J Fam Pract. 2009; 58(11 Suppl Postmenopausal):S1-S6.

6. van Geel TA, Geusens PP, Winkens B, Sels JP, Dinant GJ. Measures of bioavailable serum testosterone and estradiol and their relationships with muscle mass, muscle strength and bone mineral density in postmenopausal women: a cross-sectional study. Eur J Endocrinol. 2009;160(4): 681-687.

7. Sprague BL, Trentham-Dietz A, Cronin KA. A sustained decline in postmenopausal hormone use: results from the National Health and Nutrition Examination Survey, 1999-2010. Obstet Gynecol. 2012;120(3): 595-603.

8. Sprague BL, Trentham-Dietz A, Remington PL. The contribution of postmenopausal hormone use cessation to the declining incidence of breast cancer. Cancer Causes Control. 2011;22(1):125-134. 
9. Weiss NS, Treggiari MM. Postmenopausal hormone use and skeletal fracture: does the size of the benefit decrease with increasing age? Obstet Gynecol. 2002;100(2):364-368.

10. Madsen OR, Schaadt O, Bliddal H, Egsmose C, Sylvest J. Relationship between quadriceps strength and bone mineral density of the proximal tibia and distal forearm in women. J Bone Miner Res. 1993;8(12): 1439-1444.

11. Taaffe DR, Pruitt L, Lewis B, Marcus R. Dynamic muscle strength as a predictor of bone mineral density in elderly women. J Sports Med Phys Fitness. 1995;35(2):136-142.

12. Graves JE, Pollock ML, Carroll JF. Exercise, age, and skeletal muscle function. South Med J. 1994;87(5):S17-S22.

13. Brown LE. Isokinetics in Human Performance. Lower Mitcham, SA, Australia: Human Kinetics; 2000.

14. von Stengel S, Kemmler W, Kalender WA, Engelke K, Lauber D. Differential effects of strength versus power training on bone mineral density in postmenopausal women: a 2-year longitudinal study. $\mathrm{Br} J$ Sports Med. 2007;41(10):649-655; discussion 655.

15. World Health Organization. Life expectancy from birth. 2012. Available from: http://apps.who.int/gho/athena/data/GHO/ WHOSIS_000001,WHOSIS_000015.xml?filter=COUNTRY:*;REGI ON:AFR. Accessed September 20, 2013.

16. Kannus $P$. Isokinetic evaluation of muscular performance: implications for muscle testing and rehabilitation. Int J Sports Med. 1994;15 Suppl 1: S11-S18.

17. Humphries B, Triplett-McBride T, Newton RU, et al. The relationship between dynamic, isokinetic and isometric strength and bone mineral density in a population of 45 to 65 year old women. J Sci Med Sport. 1999;2(4):364-374.

18. Sherk VD, Palmer IJ, Bemben MG, Bemben DA. Relationships between body composition, muscular strength, and bone mineral density in estrogen-deficient postmenopausal women. J Clin Densitom. 2009;12(3):292-298.
19. Peterson MC, Riggs MM. Predicting nonlinear changes in bone mineral density over time using a multiscale systems pharmacology model. CPT Pharmacometrics Syst Pharmacol. 2012;1:e14.

20. Nakazawa K, Kawakami Y, Fukunaga T, Yano H, Miyashita M. Differences in activation patterns in elbow flexor muscles during isometric, concentric and eccentric contractions. Eur J Appl Physiol Occup Physiol. 1993;66(3):214-220.

21. Blain H, Vuillemin A, Teissier A, Hanesse B, Guillemin F, Jeandel C. Influence of muscle strength and body weight and composition on regional bone mineral density in healthy women aged 60 years and over. Gerontology. 2001;47(4):207-212.

22. Marin RV, Pedrosa MA, Moreira-Pfrimer LD, Matsudo SM, LazarettiCastro M. Association between lean mass and handgrip strength with bone mineral density in physically active postmenopausal women. J Clin Densitom. 2010;13(1):96-101.

23. Iki M, Saito Y, Dohi Y, et al. Greater trunk muscle torque reduces postmenopausal bone loss at the spine independently of age, body size, and vitamin D receptor genotype in Japanese women. CalcifTissue Int. 2002;71(4):300-307.

24. Gerdhem P, Ringsberg KA, Akesson K, Obrant KJ. Influence of muscle strength, physical activity and weight on bone mass in a populationbased sample of 1004 elderly women. Osteoporos Int. 2003;14(9): $768-772$.

25. Babyak MA. What you see may not be what you get: a brief, nontechnical introduction to overfitting in regression-type models. Psychosom Med. 2004;66(3):411-421.

26. Iki M, Saito Y, Kajita E, Nishino H, Kusaka Y. Trunk muscle strength is a strong predictor of bone loss in postmenopausal women. Clin Orthop Relat Res. 2006;443:66-72.
Clinical Interventions in Aging

\section{Publish your work in this journal}

Clinical Interventions in Aging is an international, peer-reviewed journal focusing on evidence-based reports on the value or lack thereof of treatments intended to prevent or delay the onset of maladaptive correlates of aging in human beings. This journal is indexed on PubMed Central, MedLine, the American Chemical Society's 'Chemical Abstracts

\section{Dovepress}

Service' (CAS), Scopus and the Elsevier Bibliographic databases. The manuscript management system is completely online and includes a very quick and fair peer-review system, which is all easy to use. Visit http://www.dovepress.com/testimonials.php to read real quotes from published authors. 